\title{
Real-Space Evidence of Trimeric, Tetrameric, and Pentameric Uracil Clusters Induced by Alkali Metals
}

\section{Electronic supplementary information}

Yuanqi Ding, Xinyi Wang, Donglin $\mathrm{Li}$ and Wei Xu*

Interdisciplinary Materials Research Center, College of Materials Science and Engineering,

Tongji University, Shanghai 201804, P. R. China

E-mail: xuwei@tongji.edu.cn 


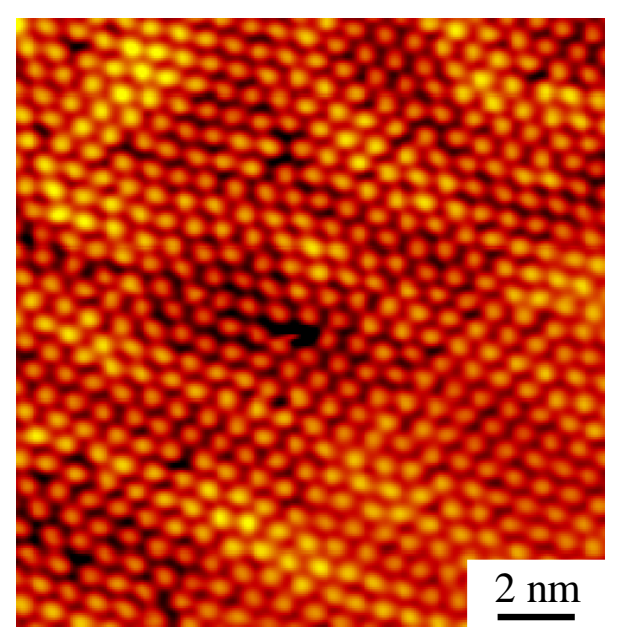

Figure S1. The STM image of the close-packed structure formed by pure uracil on the $\mathrm{Au}(111)$ surface by annealing at $350 \mathrm{~K}$ without addition of alkali metals. Scanning conditions: $I_{t}=-0.8 \mathrm{nA}$, $V_{t}=-1.4 \mathrm{~V}$.

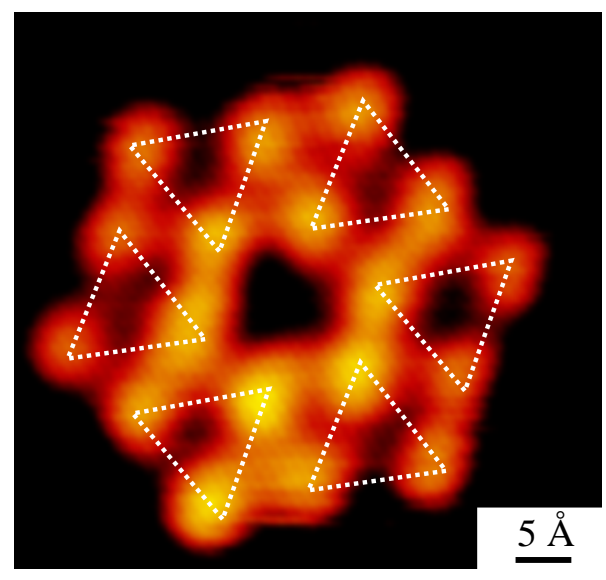

Figure S2. A close-up STM image of the hexagon structure with exaggerated contrast to show the dim center in the triangular clusters. Scanning conditions: $I_{t}=1.2 \mathrm{nA}, V_{t}=1.7 \mathrm{~V}$. 


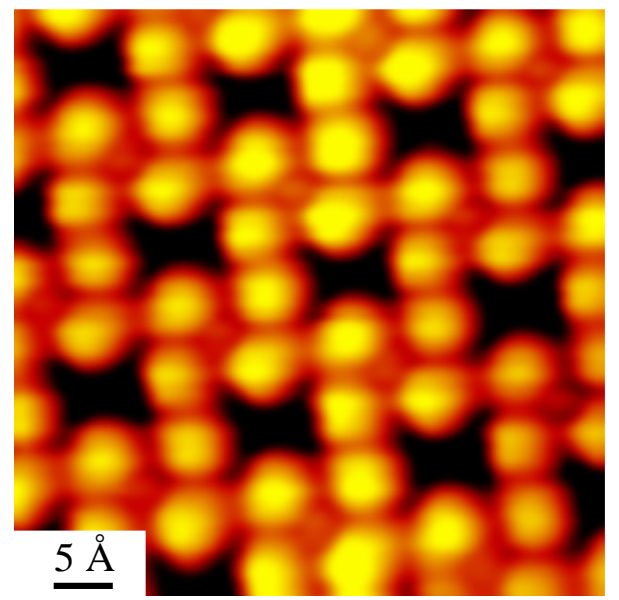

Figure S3. A close-up STM image of the $\mathrm{U}_{4} \mathrm{Na}$ network with a special tip state in which the central Na can be visible. Scanning conditions: $I_{t}=-0.6 \mathrm{nA}, V_{t}=-1.4 \mathrm{~V}$. 